\title{
Taste loss in hospitalized multimorbid elderly subjects
}

This article was published in the following Dove Press journal:

Clinical Interventions in Aging

12 February 2013

Number of times this article has been viewed

\section{ED Toffanello' \\ EM Inelmen' \\ A Imoscopi \\ E Perissinotto ${ }^{2}$ \\ A Coin' \\ F Miotto' \\ LM Donini ${ }^{3}$ \\ D Cucinotta ${ }^{4}$ \\ M Barbagallo ${ }^{5}$ \\ E Manzato' \\ G Sergi'}

'Department of Medical and Surgical Sciences, Geriatrics Division and University of Padova, Padova, ${ }^{2}$ Department of Environmental Medicine and Public Health, University of Padua, Padua, Italy; ${ }^{3}$ Department of Medical Physiopathology (Food Science Section), University of Roma, La Sapienza, Roma, ${ }^{4} \mathrm{~S}$ Orsola Malpighi Hospital, Bologna, ${ }^{5}$ Geriatric Unit, Department of Internal Medicine and Emerging Diseases, University of Palermo, Palermo, Italy
Correspondence: Elena D Toffanello Clinica Geriatrica, Ospedale Giustiniane, via Giustiniani 2, 35I 28, Padova, Italy Tel +390498218492

Fax +3904 982। 1218

Email elenadebora.toffanello@sanita. pasdova.it
Background: Loss of the sense of taste is common among older people. Morbidities and polypharmacy may contribute to the age-related decline in gustatory function. The aims of the present study were to investigate taste perception in elderly hospitalized patients by comparing their taste recognition thresholds with those of healthy, free-living elderly individuals and to identify potential determinants of taste loss.

Methods: The participants in this observational study were 55 elderly patients hospitalized in the acute geriatric section of the Department of Medical and Surgical Sciences at Padova University and 41 free-living individuals aged older than 65 years, randomly recruited from elderly people attending mild fitness programs at public gymnasiums in Padova. Data were collected on nutrition, health, cognitive, and functional status for all participants. Gustatory capabilities were assessed using aqueous solutions of sucrose, sodium chloride, citric acid, and quinine hydrochloride (representing sweet, salty, sour, and bitter stimuli, respectively), and taste recognition thresholds were measured in both groups.

Results: In comparison with the free-living elderly subjects, those in hospital were significantly less able to recognize the taste of citric acid $(P<0.05)$. Low citric acid sensitivity was independently associated with advanced age ( $\geq 75$ years; odds ratio [OR] 3.01, 95\% confidence interval [CI] 1.01-9.82), polypharmacy (number of prescribed drugs $\geq 4$; OR 2.74, 95\% CI 1.01-7.72), and poor nutritional status (as assessed by Mini Nutritional Assessment score $<23.5$; OR 5.08, 95\% CI 1.76-14.6).

Conclusion: Because gustatory impairment may reduce a person's appetite and lead to inadequate dietary intake, compensatory nutritional measures, such as the use of flavor-enhanced foods, should be strongly encouraged, particularly in the hospital setting.

Keywords: taste thresholds, taste loss, hospitalized elderly, sour stimuli, polypharmacy, malnutrition

\section{Introduction}

Loss of the sense of taste is common among older people, ${ }^{1}$ and a decline in gustatory function, due to a physiological decline in the density of the taste buds and papillae, ${ }^{2}$ is reportedly as part of the normal aging process. ${ }^{3}$ Several studies on taste dysfunction have concluded that changes in taste bud density with increasing age may affect taste function differently at different regions of the tongue. There is consequently reason to believe that sensitivity to the four basic taste qualities, ie, sweet, salty, bitter, and sour, might not be uniformly impaired in the elderly. ${ }^{4}$ Compared with younger adults, people aged 70 years and older seem to have a decreased sensitivity to sour, bitter, and salty stimuli, while their perception of sweet taste seems to be unaffected. ${ }^{5}$ 
Distorted gustatory function in older age may also be influenced by disease, functional and cognitive impairment, depression, or polypharmacy, which have all been documented as significant but underestimated contributors to taste disorders in the elderly. ${ }^{6,7}$ Elderly hospitalized subjects, who are frequently exposed to a number of debilitating diseases and drugs, might therefore be more likely to have an impaired sense of taste. The gustatory distortion in elderly subjects is thought to have several clinical consequences, eg, diminished appreciation of food and beverages, and a declining appetite, which may increase the risk of anorexia, weight loss, and malnutrition. ${ }^{3}$ Reliable data on taste perception in hospitalized elderly subjects are still lacking, and the clinical and biological features associated with taste impairment in such patients are still not clear.

We hypothesized that taste impairment would be more frequent in hospitalized than in free-living healthy elderly people. The aims of the present study were: first, to investigate whether the sense of taste among the hospitalized elderly differs in relation to certain tastants, comparing their recognition thresholds for four basic taste qualities with those of healthy free-living elderly people; and second, to analyze the associations between taste perception and clinical features in frail hospitalized older people.

\section{Materials and methods}

\section{Study design and participants}

A total of 96 subjects, comprising 55 hospitalized patients and 41 community-dwelling individuals, aged 65 years and over, with no previous experience of taste-related research, were enrolled in this study.

The hospitalized subjects ( 35 women and 20 men, aged $82.7 \pm 7.0$ years) were selected from patients consecutively admitted from April 2008 to February 2010 to the acute geriatric section of the Department of Medical and Surgical Sciences at Padua University, based on the following exclusion criteria: need for parenteral and/or enteral nutrition, severe comorbidity (grade 4 at the Cumulative Illness Rating Scale), ${ }^{8}$ severe cognitive impairment, and dysphagia.

The free-living subjects ( 27 women and 14 men, aged $74.5 \pm 4.6$ years) were randomly recruited on a voluntary basis from elderly people attending a twice-weekly mild fitness program at public gymnasiums in Padova. Their health was assessed by medical personnel, based on their clinical history and a clinical examination: current smokers (defined as daily or occasional smokers), heavy drinkers (defined by a daily consumption of five or more standard drinks, each one containing 14 grams of pure alcohol in men, and four or more standard drinks in women, according to the definition of the National Institute on Alcohol Abuse and Alcoholism), and individuals on chemotherapy or diagnosed with cancer were excluded.

This study was part of a Research Project of National Interest sponsored by the Italian Ministry for Education, University and Research and was approved by the local ethics committee. In accordance with the Declaration of Helsinki, all participants were fully informed about the nature of the study, and its purpose, procedures, and risks, and gave their informed consent.

\section{Measures}

Data were obtained on all participants' nutritional, health, cognitive and functional status by trained personnel at the Geriatrics Department of Padova University. Nutritional status was assessed by:

- Serum albumin level (g/dL), for which fasting blood samples were obtained by venipuncture and then processed, aliquoted, and frozen at the Padova University laboratory for diagnostic analysis.

- Anthropometric measures, ie, height, weight, mid arm circumference, and calf circumference, were measured with subjects' barefoot and wearing light clothes. ${ }^{9}$ For patients unable to stand up, height was estimated using the knee-heel length according to Chumlea's technique. ${ }^{10}$ Body mass index $\left(\mathrm{kg} / \mathrm{m}^{2}\right)$ was then calculated.

- The Mini Nutritional Assessment (MNA) questionnaire, an internationally validated tool which consists of 18 items, including data on anthropometric measures, health status, dietary patterns, and subjective assessments of the participant's nutritional and health status. A total score $\geq 23.5$ distinguishes patients with good nutritional status from those at nutritional risk (MNA score, 17-23.5) and those with protein-calorie malnutrition (MNA score $<17$ ). ${ }^{11}$

Functional and cognitive status were assessed using the activity of daily living tool, the Instrumental Activities of Daily Living (IADL) scale, ${ }^{12,13}$ according to Lawton and Brody ${ }^{14}$ and the Mini Mental State Examination (MMSE) devised by Folstein et al, ${ }^{15}$ in which scores range from 0 to 30 . The crude MMSE score was adjusted for age and formal education using the coefficients proposed for the Italian population; ${ }^{16}$ participants with severe cognitive impairment (MMSE adjusted score $<18$ ) were not enrolled. Depressive symptoms were assessed using the short form of the Geriatric Depression Screening (GDS) scale. ${ }^{17}$ 
Health status was assessed using the Cumulative Illness Rating Scale (CIRS), ${ }^{8}$ which evaluates comorbidities and illness severity levels, classifying comorbidities for 13 organ systems and grading each condition from 0 (no problem) to 4 (severely incapacitating or life-threatening conditions). The CIRS comorbidity index is given by the number of conditions graded as $\geq 3 .{ }^{8}$ The number of drugs prescribed was also considered and polypharmacy was defined as the consumption of four or more different medications, even of the same chemical class. ${ }^{18}$

\section{Taste functions}

Solutions representing the four basic tastes (sweet, salty, sour, and bitter) were prepared by trained personnel using sucrose, sodium chloride, citric acid, and quinine hydrochloride, respectively. Each compound was presented in four different concentrations, for a total of 16 aqueous solutions of sucrose $(0.0032,0.010,0.032,0.10 \mathrm{M})$, sodium chloride $(0.0032,0.010,0.032,0.10 \mathrm{M})$, citric acid $(0.00037,0.0011$, $0.0037,0.011 \mathrm{M})$, and quinine hydrochloride $(1.0,3.2,10$, $32 \mathrm{mM}$ ). All solutions were prepared using analytical grade reagents in deionized water and stored at $4{ }^{\circ} \mathrm{C}$ in screw-capped glass bottles for no longer than one week to preserve their taste qualities. The taste functions were assessed using an ascending-series staircase methodology. ${ }^{13}$ The task was a two alternative (test stimulus and control), forced-choice, single series procedure, with concentrations presented in ascending order as described elsewhere, ${ }^{13}$ and based on the method used by Mojet et al. ${ }^{19}$

In each trial, a participant received two $30 \mathrm{~mL}$ disposable plastic cups, one containing $10 \mathrm{~mL}$ of taste solution and one containing $10 \mathrm{~mL}$ of distilled water, previously brought up to room temperature $\left(22^{\circ} \mathrm{C}-25^{\circ} \mathrm{C}\right)$. The two cups were placed side by side in front of the subjects in a left-right position randomly chosen from trial to trial. The sip and spit method was used. At the start of the section and before each trial, the subject rinsed with distilled water and expectorated. The samples, both the blanks and taste solutions, were swirled around the mouth briefly and spat out. After indicating which cup contained the compound, the participant received another pair of samples. Whenever the subject selected incorrectly, the next trial took place at the next higher step. When the subjects selected correctly, the same concentration was presented again. If a given taste was not recognized at any trials, the result of the test was recorded as "scale out". The lowest concentration at which participants correctly identified the taste was recorded as their recognition threshold, which could range from the lower concentrated solution, numbered as 1 , to the higher concentrated solution, numbered as 4 . Reduced taste perception was defined as the inability to recognize a taste at the concentrations numbered $\leq 2$, according to the results for taste thresholds obtained from a large population of healthy normal-eating free-living elderly individuals, previously enrolled in the multicentric project of which this study formed part. ${ }^{13}$

\section{Statistical analysis}

Normality of distribution was tested with Shapiro-Wilk statistics. Results were expressed as the mean \pm standard deviation for normally distributed variables, and as the median with first and third quartiles for non-normally distributed variables. Categorical variables were described as frequency distributions. Differences between free-living and hospitalized elderly subjects were tested using the Student's unpaired $t$-test or analysis of variance for normally distributed variables and the non-parametric Kruskal-Wallis test for non-normally distributed variables. The Chi-square test was applied to compare categorical distributions. Comparisons of mean recognition thresholds between the inpatients and free-living people were drawn using analysis of variance, and Tukey's method for multiple comparisons was applied. Differences between groups in the percentages of subjects with reduced taste perception were assessed by logistic regression analysis adjusting for age and interaction term (hospitalization*age). All comparisons between groups were adjusted for age, by the means of linear regression analysis for continuous variables and logistic regression analysis for categorical ones. For each compound, the relationship between reduced taste perception, as the dependent variable (defined as the inability to recognize the taste at concentrations numbered 1 or 2), and several clinical/biological features was assessed using separate simple logistic regression analysis performed on the sample as a whole. The covariates considered in the analysis were age, body mass index [BMI], CIRS comorbidity index, serum albumin levels, number of prescribed drugs, and GDS, MMSE, and MNA scores. The logistic regression analysis were applied by entering covariates one by one, both as quantitative and dichotomous/ranked. Age was dichotomized on the first quartile of the sample distribution and categorized as $65-75$ years and over 75 years; BMI was classified in two categories, ie, normal weight (20-24.9 kg/m $)$, and overweight/obese $\left(\geq 25 \mathrm{~kg} / \mathrm{m}^{2}\right)$. Only four subjects in our sample were underweight $(\mathrm{BMI}<20)$. The BMI threshold of $20 \mathrm{~kg} / \mathrm{m}^{2}$ has been commonly accepted as defining underweight in elderly people. ${ }^{20}$ For the present study, a BMI $\geq 25 \mathrm{~kg} / \mathrm{m}^{2}$ was assumed as a reference category, 
because overweight/obese status has been associated with a better taste recognition ability in the elderly. ${ }^{21}$ Adjusted odds ratios (OR) and 95\% confidence intervals (CI) were estimated. Variables significantly associated with reduced taste perception were entered in the multiple logistic regression models and adjusted OR with 95\% CI were estimated on the whole sample population with "group" (hospitalization) as a confounder, also evaluating for the interaction term (hospitalization*age). The level of statistical significance was set at 0.05. All statistical analysis was performed using SAS software, release 9.2 (SAS Institute, Inc, Cary, NC).

\section{Results}

\section{Clinical features}

The general characteristics of the free-living and hospitalized participants are outlined in Table 1 . There were no significant differences in gender composition between the two groups (Chi-square test, $P=0.49$ ). The hospitalized subjects were significantly older than the free-living elderly subjects and had a lower mean BMI. Most of the hospitalized subjects were normal-weight or overweight (36\% and $42 \%$, respectively, details not shown). The hospitalized elderly were significantly more disabled, depressed, and cognitively impaired.
For the inpatients, total MNA scores were lower, whereas comorbidity index and number of prescribed drugs were significantly higher. The community-dwelling participants were almost all fully independent in activities of daily living, functioned well, and had no diseases interfering with their well-being or nutritional status.

\section{Taste functions}

Figure 1 shows the cumulative percentage of distribution of the free-living and hospitalized elderly subjects according to their recognition thresholds for quinine, sodium chloride, citric acid, and sucrose. The pattern of the curves for quinine, sodium chloride, and sucrose was similar in the two groups, suggesting that free-living and hospitalized subjects did not differ in their ability to recognize bitter, salty, and sweet stimuli. For citric acid taste, the slope of the curve was markedly flatter for the hospitalized patients, indicating that sour recognition thresholds were significantly higher in hospitalized than in healthy elderly people $(P=0.002$, Chi-square test for differences between groups).

Figure 2 shows the mean recognition thresholds for the four basic taste qualities in the two groups: hospitalized subjects needed higher concentrations in order to

Table I Characteristics of free-living and hospitalized elderly subjects

\begin{tabular}{|c|c|c|c|c|}
\hline & $\begin{array}{l}\text { Free-living } \\
n=4 I\end{array}$ & $\begin{array}{l}\text { Hospitalized } \\
n=55\end{array}$ & $P$ value* & $\begin{array}{l}\text { Age-adjusted } \\
P \text { value }\end{array}$ \\
\hline Gender, \% (F) & $65.8 \%$ & $63.6 \%$ & 0.49 & 0.52 \\
\hline Age, (years) & $74.5 \pm 4.6$ & $82.7 \pm 7.0$ & $<0.000$ I & - \\
\hline BMI, $\left(\mathrm{kg} / \mathrm{m}^{2}\right)$ & $28.8 \pm 3.9$ & $25.5 \pm 4.1$ & 0.0002 & 0.05 \\
\hline$<25$ & $19.5 \%$ & $44 \%$ & 0.01 & 0.26 \\
\hline$\geq 25$ & $80.5 \%$ & $56 \%$ & & \\
\hline Mid-arm circumference, $(\mathrm{cm})$ & $26.9 \pm 3.3$ & $23.2 \pm 3.0$ & $<0.0001$ & 0.85 \\
\hline Calf circumference, $(\mathrm{cm})$ & $37.7 \pm 2.9$ & $32.6 \pm 3.9$ & $<0.000 \mathrm{I}$ & 0.004 \\
\hline ADL disability, ( $>2$ functions lost) & $0 \%$ & $23.6 \%$ & $<0.001$ & 0.04 \\
\hline IADL disability, (score $<$ II) & $2.4 \%$ & $54.5 \%$ & $<0.0001$ & 0.001 \\
\hline GDS score, $(0-15)$ & $3(I-4)$ & $4(2-7)$ & 0.003 & 0.15 \\
\hline Depression, \% & $7.3 \%$ & $30 \%$ & 0.0052 & 0.20 \\
\hline MMSE score, $(0-30)$ & $28.3(27.3-29)$ & $24(23-27)$ & $<0.000 \mathrm{I}$ & 0.009 \\
\hline Cognitive impairment, $\%$ & $2.44 \%$ & $38.8 \%$ & $<0.0001$ & 0.02 \\
\hline MNA score, $(0-30)$ & $27.5(26-28.5)$ & $20(18-25)$ & $<0.0001$ & $<0.0001$ \\
\hline Risk of malnutrition, $\%$ & $2.4 \%$ & $74.5 \%$ & $<0.0001$ & $<0.0001$ \\
\hline Comorbidity index & I (0-2) & $3(2-4)$ & $<0.0001$ & $<0.0001$ \\
\hline Severity index & $\mathrm{I} .4 \pm 0.3$ & $1.9 \pm 1.8$ & $<0.000$ I & $<0.0001$ \\
\hline Number of prescribed drugs & $2(I-4)$ & $6(4-8)$ & $<0.0001$ & $<0.0001$ \\
\hline Albumin serum level (g/dL) & $4.3 \pm 0.2$ & $3.3 \pm 0.6$ & $<0.0001$ & $<0.0001$ \\
\hline
\end{tabular}

Notes: Frequencies of distribution are expressed as percentages. Means and standard deviations are used for normally distributed variables; medians and first and third quartiles are used for non-normally distributed variables. *Comparisons between groups were done using analysis of variance for normally distributed variables and the nonparametric Kruskal-Wallis test for non-normally variables. The Chi-square test was used to compare categorical data. Age-adjusted $P$ values were obtained from the mean of linear regression analysis for quantitative variables and by logistic regression analysis for categorical ones.

Abbreviations: ADL, activities of daily living (disability was defined as two or more functions lost); IADL, Instrumental Activities of Daily Living (disability defined by a score > II); GDS, Geriatric Depression Scale (score range 0-15, higher scores indicate presence of depression); MMSE, Mini Mental Examination (score range 0-30, higher scores represent better cognitive function); MNA, Mini Nutritional Assessment (score range 0-30, with highest values representing better nutritional status). 


\section{Dotted lines (----) free-living; Solid lines (-) hospitalized}

\section{A Quinine}

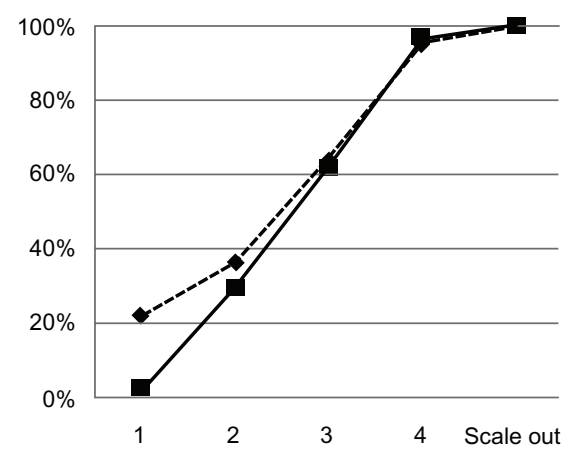

C Citric acid

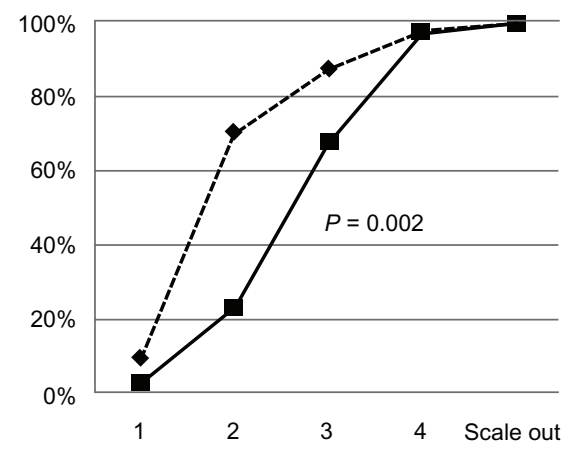

B Sodium chloride

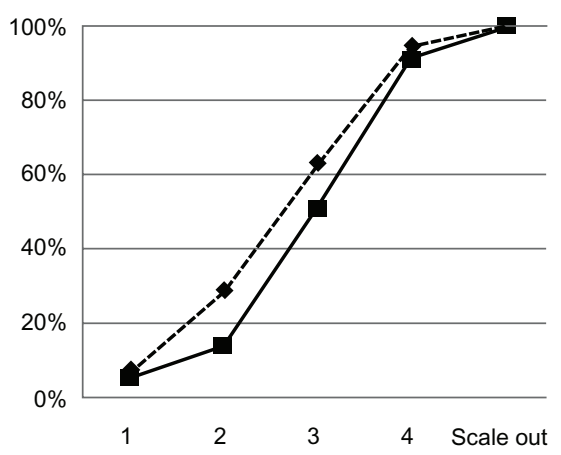

D Sucrose

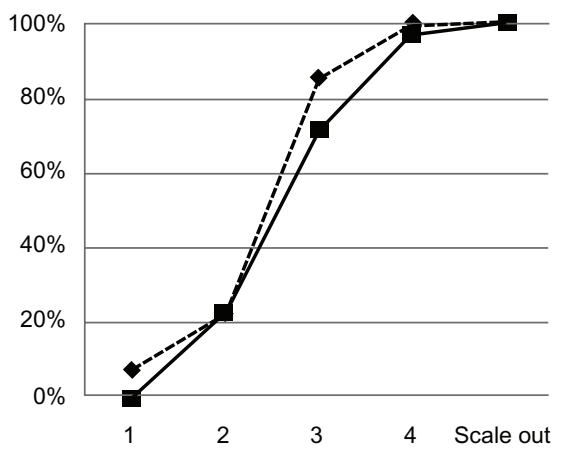

Figure I Cumulative percentage of distribution of hospitalized and free-living subjects, according to their recognition thresholds, for the four basic taste qualities. Notes: Concentrations of solution were numbered in ascending order from I to 4; "scale out" refers to subjects who failed to recognize the taste at any concentration. Differences in distribution between groups were assessed using the Chi-squared test.

recognize each of the four tastes, although the difference between groups was only statistically significant for citric acid $(P=0.01)$. The percentage of participants with a reduced taste perception for the four basic taste qualities is shown in Figure 3. Compared with the free-living elderly,

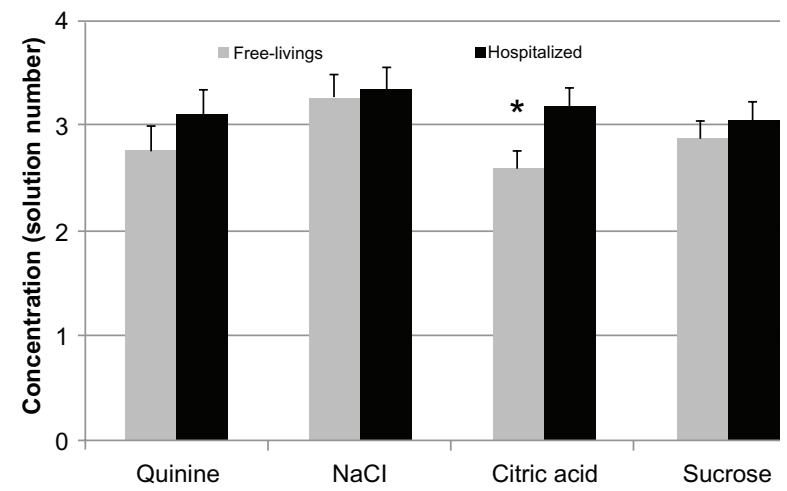

Figure 2 Recognition thresholds of free-living and hospitalized subjects for the four basic taste qualities.

Notes: Values are given as mean solution number and standard error. Asterix indicates a significant difference $(P=0.01)$ between the mean thresholds for hospitalized and free-living elderly subjects. Differences between groups were assessed using analysis of variance, adjusting for age and for multiple comparisons with Tukey's method. the hospitalized patient group tended to have a higher prevalence of individuals unable to recognize the taste of the solutions at lower concentrations, although the two groups differed to a statistically significant degree only in the case of citric acid $(P=0.0002)$.

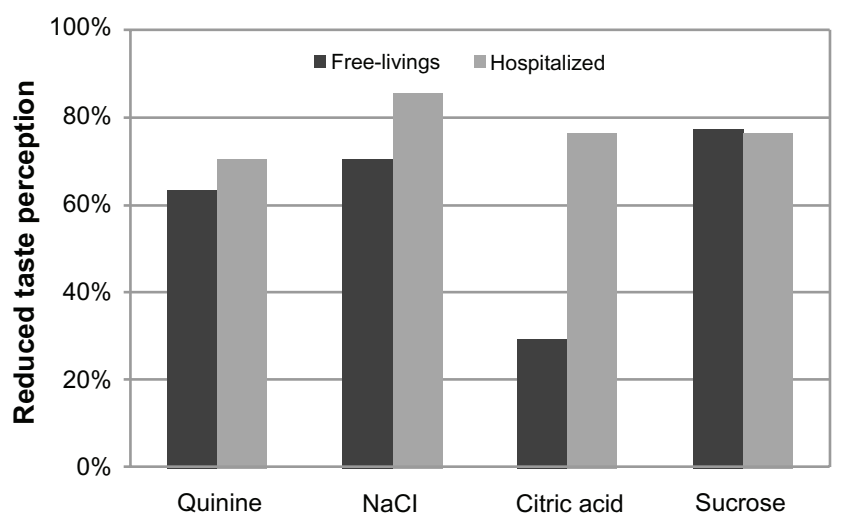

Figure 3 Percentage of free-living and hospitalized subjects with a reduced taste perception for the four basic tastes.

Notes: Differences between groups were tested using simple logistic regression analysis, considering reduced taste perception (inability to recognize the taste at lower concentrations) as the dependent variable, and adjusting for age and interaction effect (hospitalization*age). 
Simple logistic regression analysis was conducted for each test solution in the sample as a whole. Statistically significant associations with recognition thresholds were only seen for citric acid (Table 2) and not for the other three tastes. A significant association emerged between advanced age ( $>75$ years) and reduced citric acid perception (OR 4.55, CI 95\% 1.53-13.41). After adjusting for age, the reduced sour perception was associated, in the whole group, with scores obtained on the GDS, MMSE, and MNA, and with number of drugs. The results of the logistic regression analysis did not change when covariates were entered as quantitative rather than dichotomous/ranked (details not shown).

Table 3 shows the results of the multiple logistic regression analysis used to assess the independent effect of the associated variables on reduced sour taste perception in the sample as a whole. Advanced age ( $>75$ years) and polypharmacy ( $\geq$ four drugs) were positively related to an increased risk of reduced sour taste perception. When malnutrition risk (MNA score $<23.5$ ) was entered in the model, this remain the only covariate associated with a significantly increased risk (OR 3.6, 95\% CI 1.3-11.4, $P=0.03$ ) of a poor ability to recognize citric acid. Similar results were obtained even considering the covariates as continuous variables (details not shown). Forcing into the analysis the confounding effect of the "group" (hospitalization: yes/no) and the interaction

Table 2 Association of reduced citric acid perception with clinical and biological features

\begin{tabular}{|c|c|c|c|}
\hline Variables & $\begin{array}{l}\text { Odds } \\
\text { ratio }\end{array}$ & $95 \% \mathrm{Cl}$ & $\begin{array}{l}\text { Age-adjusted } \\
P \text { value }\end{array}$ \\
\hline Age $<75$ years & 1 & - & - \\
\hline$\geq 75$ years & 4.55 & $|.53-13.4|$ & 0.006 unadjusted \\
\hline $\mathrm{BMI}<25 \mathrm{~kg} / \mathrm{m}^{2}$ & 2.63 & $0.88-7.89$ & 0.08 \\
\hline$\geq 25 \mathrm{~kg} / \mathrm{m}^{2}$ & I & - & - \\
\hline ADL score & 0.97 & $0.79-1.18$ & 0.77 \\
\hline IADL score & 0.89 & $0.75-1.04$ & 0.15 \\
\hline $\begin{array}{l}\text { Depression } \\
\text { (GDS score } \geq 6 \text { ) }\end{array}$ & 4.08 & $0.95-|7.5|$ & 0.05 \\
\hline $\begin{array}{l}\text { Cognitive impairment } \\
(M M S E \leq 24)\end{array}$ & 4.47 & $1.10-18.16$ & 0.03 \\
\hline $\begin{array}{l}\text { Malnutrition/risk } \\
\text { for malnutrition } \\
(\text { MNA }<23.5)\end{array}$ & 3.20 & $1.04-9.90$ & 0.04 \\
\hline Comorbidity index & 1.20 & $0.88-1.6 \mid$ & 0.23 \\
\hline $\begin{array}{l}\text { Albumin serum level } \\
(<3.5 \mathrm{~g} / \mathrm{dL})\end{array}$ & 3.30 & $0.93-12.10$ & 0.06 \\
\hline Polypharmacy ( $\geq 4$ drugs) & 2.83 & $1.00-7.99$ & 0.04 \\
\hline
\end{tabular}

Note: Simple regression analysis adjusted for age in the sample as a whole.

Abbreviations: BMI, body mass index $\left(\mathrm{kg} / \mathrm{m}^{2}\right)$; $\mathrm{ADL}$, activities of daily living (score range $0-12$, highest values indicate functional dependency); $\mathrm{Cl}$, confidence interval; IADL, Instrumental Activities of Daily Living (score range 0-14, highest values indicate highest degree of disability); GDS, Geriatric Depression Scale score; MMSE, Mini Mental State Examination score; MNA, Mini Nutritional Assessment score.
Table 3 Results of logistic regression analysis, using stepwise selection, on significant independent factors, associated with reduced citric acid perception

\begin{tabular}{|c|c|c|c|}
\hline Independent variables & $\begin{array}{l}\text { Odds } \\
\text { ratio }\end{array}$ & $95 \% \mathrm{Cl}$ & $P$ values \\
\hline \multicolumn{4}{|l|}{ §Model I } \\
\hline Age $\geq 75$ years & 3.06 & $1.0-9.9$ & 0.05 \\
\hline $\begin{array}{l}\text { Polypharmacy ( } \geq 4 \text { drugs) } \\
\S \S \text { Model } 2\end{array}$ & 2.67 & I.0-7.7 & 0.05 \\
\hline $\begin{array}{l}\text { Malnutrition/risk of } \\
\text { malnutrition }(\text { MNA }<23.5) \\
\text { \$§\$Model } 3\end{array}$ & 2.9 & $1.0-9.6$ & 0.05 \\
\hline Hospitalization & 7.9 & $2.74-22.8$ & 0.0001 \\
\hline
\end{tabular}

Notes: ${ }^{\S}$ Variables entered in model I: age $>75$ years, depression (GDS score $\geq 6$ ), cognitive impairment (MMSE score $\leq 24$ ), polypharmacy (number of drugs $\geq 4)$; ${ }^{\$ 6}$ variables entered in model 2 : variables in model I plus malnutrition risk (MNA < 23.5); ${ }^{\S \S}$ variables entered in model 3: variables entered in model I and 2 plus covariate of hospitalization (yes/no) and interaction effect (hospitalization*age).

Abbreviations: $\mathrm{Cl}$, confidence interval; GDS, Geriatric Depression Scale score; MMSE, Mini Mental State Examination score; MNA, Mini Nutritional Assessment score.

term, being hospitalized was associated with an increased risk (OR 7.9, 95\% CI 2.7-11.4, $P=0.0001$ ) of poor ability to recognize sour taste.

\section{Discussion}

The results of the present study support the hypothesis that an impaired sense of taste might be more frequent among the hospitalized elderly, but their ability to identify the four basic taste qualities does not seem to be uniformly impaired by comparison with the sense of taste among healthier, free-living elderly subjects. It might be expected that factors such as advanced age, multidrug therapy, comorbidities and malnutrition, conditions which are frequently observed in the frail hospitalized elderly, might be responsible for an overall reduction in taste perception and gustatory ability. However, as evidenced by our findings, sensitivity to sour taste seemed to be the only one affected in our hospitalized elderly patients, while their recognition thresholds for bitter, salty, and sweet tastes did not differ significantly from that of healthier, free-living elderly subjects.

The results of our study highlight that disease status and medications contribute to the well-known age-related taste losses, mainly by altering the ability to perceive sour taste, without interfering with the ability to perceive other tastants.

To our knowledge, only one study has documented a reduced sour perception in frail elderly hospitalized patients, but the sample consisted of only 15 subjects. ${ }^{22}$ In another study, which included 100 hospitalized subjects, the mean age of participants was 56 years and the taste loss documented was not related to a specific taste. ${ }^{7}$ 
It has been recognized that gustatory decline commonly starts towards the end of the sixth decade and becomes more pronounced before 75 years of age. ${ }^{4}$ Although the design of the present study did not envisage a control group of younger adults, the mean recognition thresholds recorded in our elderly subjects were similar to those reported in a previous study of old people, ${ }^{23}$ and were significantly higher than those reported for a control group of younger adults (aged 18-25 years), except for the sucrose recognition thresholds, which did not differ between the two age groups. These findings suggest that age affects a person's sense of sour, salty, and bitter taste perception, but this does not apply to sweet taste. The age-related gustatory decline documented in our study seems to persist even in old-old subjects ( $>75$ years) but only for sour taste, as reported elsewhere. ${ }^{22}$ Reduction in sour perception also seemed to be related to disease status and drug consumption, as observed in previous research. ${ }^{22,24-26}$

A reduced sense of taste is a common side effect of many drugs, and a larger sample would have been needed to identify the relationship between any one of these drugs and the patients' taste thresholds. However, it has been well documented that side effects increase exponentially when the number of drugs taken rises to four or more. ${ }^{27}$ Supratherapeutic drug concentrations in plasma resulting from drug interactions can also alter taste cell biochemistry and/or modify other aspects of the taste pathways, ${ }^{6}$ so regardless of the types of medication involved, polypharmacy is likely to affect gustatory function, as reported previously. ${ }^{7,22}$

It is well known that critically ill elderly patients in hospitals and nursing homes consume on average from four to nine drugs. ${ }^{27}$ Hundreds of medications, including all major drug classes, have been associated clinically with taste complaints such as "loss of taste", "altered taste", and "metallic taste", ${ }^{28}$ so limiting the number of prescribed drugs might be an insufficient strategy to avoid taste perception losses in frail elderly hospitalized or institutionalized subjects.

Like advanced age and polypharmacy, nutritional status also seems to be related to gustatory function. An impaired sense of taste has been associated with loss of appetite, leading to anorexia, weight loss, and malnutrition. ${ }^{4}$ Vice versa, a poor nutritional status might induce a decline in gustatory capacity. ${ }^{6}$ The MNA tool used in the present study to assess our participants' nutritional status was shown to correlate with several outcomes, including mortality from all causes, hospital stay, and comorbidity, ${ }^{29}$ and identifying frailer elderly patients..$^{30}$ To our knowledge, ours is the first study to document an association between malnutrition, or even only the risk of malnutrition, and reduced sensitivity to sour taste. Therefore, further studies are needed to clarify the real nature of this relationship.

In the multiple logistic regression models, when the "group" effect (hospitalization: yes/no) was forced in the analysis as a confounder covariate, hospitalization remained the only variable strongly associated with risk of reduced taste sour recognition. This result does not suggest that admission to an acute geriatric ward represents a risk factor in itself, but it might be possible that being hospitalized sums up the main risk factors for sour perception impairment, ie, advanced age, drug to drug interactions, and poor nutritional status.

The main weakness of our study lies in the fact that the sample cannot be regarded as representative of the general population. Potential selection biases particularly concern the age distribution, given that our hospitalized elderly were significantly older than the free-living control group. The study design did not include a control group of younger subjects or consider the role of olfaction in gustatory properties. ${ }^{31}$ Moreover, we do not have data on recognition thresholds for the "umami" taste, which has been recognized as the fifth basic taste, as well as sour, sweet, bitter, and salty. Monosodium glutamate has a unique taste quality which is called "umami" in the Japanese language. There is no analogous word to describe this taste quality in the Italian language. Umami taste preference has been shown to be affected by nutritional status, and poorly nourished subjects prefer foods with a higher monosodium glutamate concentration than do well nourished subjects..$^{32}$ Nevertheless, the results of our study derive from strict methods for testing gustatory capabilities, and a broad array of tests to measure nutritional, health, and cognitive status that have been standardized for older people.

In conclusion, advanced age, polypharmacy, and malnutrition, which are conditions frequently observed in acute geriatric settings, seem to be strongly related to a reduced perception of sour taste, whereas the perception of the other basic tastes seems not to differ significantly from that of healthy, free-living elderly subjects. Given that age-related taste losses cannot be avoided, and limiting the number of prescribed drugs seems to be an unrealistic strategy for preventing taste complaints, much more effort is needed to promote policies improving nutritional status in hospital settings.

Commercial flavors and monosodium glutamate added to food during and after preparation of hospital meals seem to enhance smell and taste properties, improve food palatability 
and acceptance, increase salivary flow, immunity, plasma protein levels, and grip strength, and reduce oral complaints in critically ill elderly patients. ${ }^{33,34}$ Given that monosodium glutamate has been shown to add an additional taste without enhancing any other tastes, amplification of flavor and taste (with monosodium glutamate) might be regarded as a useful clinical practice to be adopted in geriatric settings.

\section{Disclosure}

The authors report no conflicts of interest in this work.

\section{References}

1. Fukunaga A, Uematsu H, Sugimoto K. Influences of aging on taste perception and oral somatic sensation. J Gerontol A Biol Sci Med Sci. 2005;60:109-113.

2. Miller IJ Jr. Variation in human taste bud density as a function of age. Ann NY Acad Sci. 1989;561:307-319.

3. Schiffman SS. Taste and smell losses in normal aging and disease. JAMA. 1997;278:1357-1362.

4. Nordin S, Brämerson A, Bringlöv E, et al. Substance and tongue-region specific loss in basic taste-quality identification in elderly adults. Eur Arch Otorhinolaryngol. 2007;264:285-289.

5. Nordin S, Razani LJ, Markison S, Murphy C. Age-associated increases in intensity discrimination for taste. Exp Aging Res. 2003;29:371-381.

6. Schiffman SS. Effects of aging on the human taste system. Ann NY Acad Sci. 2009;1170:725-729.

7. Kettaneh A, Pariès J, Stirnemann J, et al. Clinical and biological features associated with taste loss in internal medicine patients. A cross-sectional study of 100 cases. Appetite. 2005;44:163-169.

8. Parmelee PA, Thuras PD, Katz IR, Lawton MP. Validation of the Cumulative Illness Rating Scale in a geriatric residential population. J Am Geriatr Soc. 1995;43:130-137.

9. Lee RD, Nieman DC. Assessment of the hospitalized patient. In: Lee RD, Nieman DC, editors. Nutritional Assessment, 3rd ed. New York, NY: McGraw-Hill Higher Education; 2003.

10. Chumlea WC, Roche AF, Steinbaugh ML. Estimating stature from knee height for persons 60 to 90 years of age. J Am Geriatr Soc. 1995;33: 116-1120.

11. Vellas B, Guigoz Y, Baumgartner M, et al. Relationships between nutritional markers and the mininutritional assessment in 155 older persons. J Am Geriatr Soc. 2000;48:1300-1309.

12. Katz S, Downs TD, Cash HR, Grotz RC. Progress in development of the index of ADL. Gerontologist. 1970;10:20-30.

13. Donini LM, Dominguez LJ, Barbagallo M, et al. Senile anorexia in different geriatric settings in Italy. J Nutr Health Aging. 2011;15:775-781.

14. Lawton MP, Brody EM. Assessment of older people: self-maintaining and instrumental activities of daily living. Gerontologist. 1969;9: 179-186.

15. Folstein MF, Robins LN, Helzer JE. The Mini-Mental State Examination. Arch Gen Psychiatry. 1983;40:812.
16. Magni E, Binetti G, Bianchetti R, Rozzini R, Trabucchi M. Mini-Mental State Examination: a normative study in the Italian elderly population. Eur J Neurol. 1996;3:198-202.

17. Yesavage JA, Brink TL, Rose TL, et al. Development and validation of a geriatric depression screening scale: a preliminary report. J Psychiatr Res. 1982;17:37-49.

18. Goldberg RM, Mabee J, Chan L, Wong S. Drug-drug and drug-disease interactions in the ED: analysis of a high-risk population. Am J Emerg Med. 1996;14:447-450.

19. Mojet J, Christ-Hazelhof E, Heidema J. Taste perception with age: generic or specific losses in threshold sensitivity to the five basic tastes? Chem Senses. 2001;26:845-860.

20. Sergi G, Perissinotto E, Pisent C, et al. ILSA Working Group. An adequate threshold for body mass index to detect underweight condition in elderly persons: the Italian Longitudinal Study on Aging (ILSA). $J$ Gerontol A Biol Sci Med Sci. 2005;60:866-871.

21. Simchen U, Koebnick C, Hoyer S, Issanchou S, Zunft HJ. Odour and taste sensitivity is associated with body weight and extent of misreporting of body weight. Eur J Clin Nutr. 2006;60:698-705.

22. Spitzer ME. Taste acuity in institutionalized and non-institutionalized elderly men. J Gerontol. 1988;43:71-74.

23. Ahne G, Erras A, Hummel T, Kobal G. Assessment of gustatory function by means of tasting tablets. Laryngoscope. 2000;110:1396-1401.

24. Schiffman SS. Critical illness and changes in sensory perception. Proc Nutr Soc. 2007;66:331-345.

25. Schiffman SS. The role of taste and smell in appetite and satiety: impact of chemosensory changes due to aging and drug interactions. In: Wahlqvist ML, Truswell AS, Smith R, Nestle PJ, editors. Nutrition in a Sustainable Environment. Proceedings of the XV International Congress of Nutrition, Adelaide. London, UK: Smith-Gordon; 1994.

26. Schiffman SS, Graham BG, Suggs MS, Sattely-Miller EA. Effect of psychotropic drugs on a range of taste responses in young and elderly persons. Chem Senses. 1997;22:786.

27. Sergi G, De Rui M, Sarti S, Manzato E. Polypharmacy in the elderly: can comprehensive geriatric assessment reduce inappropriate medication use? Drugs Aging. 2011;28:509-518.

28. Schiffman SS, Zervakis J. Taste and smell perception in the elderly: effect of medications and disease. Adv Food Nutr Res. 2002;44:247-346.

29. Martins CP, Correia JR, do Amaral TF. Undernutrition risk screening and length of stay of hospitalized elderly. J Nutr Elder. 2005;25:2-21.

30. Izawa S, Kuzuya M, Okada K, et al. The nutritional status of frail elderly with care needs according to the mini-nutritional assessment. Clin Nutr. 2006;25:962-967.

31. Koskinen S, Kalviainen N, Tuorila H. Perception of chemosensory stimuli and related responses to flavoured yogurts in the young and elderly. Food Qual Prefer. 2003;14:623-635.

32. Murphy C. Flavor preference for monosodium glutamate and casein hydrolisate in young and elderly persons. In: Kawamura Y, Kare MR, editors. Umami: A Basic Taste. New York, NY: Marcel Dekker; 1987.

33. Schiffman SS. Intensification of sensory properties of foods for the elderly. J Nutr. 2000;130:927S-930S.

34. Schiffman SS. Sensory enhancement of foods for the elderly with monosodium glutamate and flavors. Food Rev Int. 1998;14:321-333.
Clinical Interventions in Aging

\section{Publish your work in this journal}

Clinical Interventions in Aging is an international, peer-reviewed journal focusing on evidence-based reports on the value or lack thereof of treatments intended to prevent or delay the onset of maladaptive correlates of aging in human beings. This journal is indexed on PubMed Central, MedLine, the American Chemical Society's 'Chemical Abstracts Ser-

\section{Dovepress}

vice' (CAS), Scopus and the Elsevier Bibliographic databases. The manuscript management system is completely online and includes a very quick and fair peer-review system, which is all easy to use. Visit $\mathrm{http} / / / \mathrm{www}$. dovepress.com/testimonials.php to read real quotes from published authors. 